\title{
Lateralized Eye Use Towards Video Stimuli in Bearded Dragons (Pogona vitticeps)
}

\author{
Anna Frohnwieser ${ }^{1}$, Thomas W. Pike ${ }^{1}$, John C. Murray ${ }^{2}$, and Anna Wilkinson ${ }^{1,3^{*}}$ \\ ${ }^{1}$ School of Life Sciences, University of Lincoln, Lincoln, UK \\ ${ }^{2}$ School of Computer Science, University of Lincoln, Lincoln, UK \\ ${ }^{3}$ Wildlife Research Center, Kyoto University, Japan \\ *Corresponding author (Email: awilkinson@lincoln.ac.uk)
}

Citation - Frohnwieser, A., Pike, T. W., Murray, J. C., \& Wilkinson, A. (2017). Lateralized eye use towards video stimuli in bearded dragons (Pogona vitticeps). Animal Behavior and Cognition, 4(3), 340-348. https://doi.org/10.26451/abc.04.03.11.2017

\begin{abstract}
Lateralized eye use is thought to increase brain efficiency, as the two hemispheres process different information perceived by the eyes. It has been observed in a wide variety of vertebrate species and, in general, information about conspecifics appears to elicit a left eye preference whilst information about prey elicits the opposite. In reptiles, this phenomenon has only been investigated using live conspecifics in agonistic contexts, and so it is not clear whether it can be found when using video stimuli. Here, bearded dragons (Pogona vitticeps) were presented with videos of female conspecifics and prey that either moved or were stationary, along with a control video of an empty background. Females exhibited a left eye bias towards conspecifics but males did not; however, both sexes looked at conspecifics significantly longer than prey. Further, animals used their left eye significantly longer when viewing moving stimuli of both categories. These results suggest that, in lizards, lateralized eye use when viewing conspecifics may be controlled by sex, and strongly influenced by stimulus movement. This study, therefore, provides important insights into the role of lateralized processing in lizard perception, and sets the scene for future work investigating the role of sex on perception of conspecifics and the role of motion in lateralized eye use.
\end{abstract}

Keywords - Lateralized eye use, Perception, Bearded dragon, Video stimuli

Lateralized eye use has been observed in a wide variety of vertebrate species, including mammals (Braccini, Lambeth, Schapiro, \& Fitch, 2012; Guo, Meints, Hall, Hall, \& Mills, 2009; Racca, Guo, Meints, \& Mills, 2012; Smith, Proops, Grounds, Wathan, \& McComb, 2016; Versace, Morgante, Pulina, \& Vallortigara, 2007), birds (Martinho, Burns, Von Bayern, \& Kacelnik, 2014; McKenzie, Andrew, \& Jones, 1998; Rogers, 2012; Ventolini et al., 2005; Zucca \& Sovrano, 2008), reptiles (Bonati \& Csermely, 2011; Hews \& Worthington, 2001; Robins, Chen, Beazley, \& Dunlop, 2005), amphibians (Vallortigara, Rogers, Bisazza, Lippolis, \& Robins, 1998), and fish (Bisazza, De Santi, \& Vallortigara, 1999; Bisazza, Facchin, Pignatti, \& Vallortigara, 1998; Karenina, Giljov, \& Malashichev, 2013). In vertebrates, the right brain hemisphere (which processes information obtained by the left eye) is thought to control emotions and spatial perception, while the left hemisphere (right eye) is responsible for attention, perceptual processing and the control of motor responses (Rogers, Vallortigara, \& Andrew, 2013; Vallortigara, Chiandetti, \& Sovrano, 2011). Therefore, it has been suggested that lateralized eye use increases brain efficiency, because visual information coming from each eye is processed by different brain regions (Ghirlanda \& Vallortigara, 2004; Vallortigara \& Rogers, 2005). 
Various reptile species are known to show considerable lateralization of their behavior (Rogers et al., 2013; Vallortigara \& Rogers, 2005), with information about predators and conspecifics being more easily obtained by the left eye (Bonati, Csermely, López, \& Martín, 2010; Hews, Castellano, \& Hara, 2004; Hews \& Worthington, 2001), and information about prey being more easily obtained by the right eye (Bonati \& Csermely, 2011; Bonati, Csermely, \& Romani, 2008; Bonati, Csermely, \& Sovrano, 2013; Robins et al., 2005). These studies exclusively used live stimuli, with restrained male lizards being used to stage conspecific encounters (Hews et al., 2004; Hews \& Worthington, 2001), and live mealworms (Tenebrio molitor larvae) used as prey (Bonati \& Csermely, 2011; Bonati et al., 2008; Robins et al., 2005).

Previous research investigating conspecific displays and species recognition (Clark, Macedonia, \& Rosenthal, 1997; Macedonia \& Stamps, 1994; Ord \& Evans, 2002; Ord, Peters, Evans, \& Taylor, 2002; Van Dyk \& Evans, 2007; Yang, Phelps, Crews, \& Wilczynski, 2001) has successfully used video stimuli in studies with lizards, focusing on videos of male conspecifics displaying an agonistic visual signal (head bobbing), although one study on social learning in bearded dragons has also shown that these animals are able to imitate female conspecifics seen in videos (Kis, Huber, \& Wilkinson, 2015). Therefore, we know that lizards respond to videos of conspecifics, but it is unclear what aspects of the stimulus they are responding to and which information they use from these videos. To test this, we looked at lateralized eye use in bearded dragons (Pogona vitticeps) watching and interpreting video stimuli showing conspecifics and prey that were either moving or stationary.

Bearded dragons originate from central Australia, where they are commonly found in arid areas such as savannas and desert habitats. Their territories overlap and males show strong aggressive behavior towards unfamiliar conspecifics, which often escalates into fights, during which animals can sustain substantial injuries (Rowland, 2009). Therefore, being able to quickly and reliably perceive and respond to conspecifics is beneficial, and eye lateralization has been shown to improve this ability in other lizard species (Bonati et al., 2010; Hews et al., 2004; Hews \& Worthington, 2001). Further, bearded dragons eat a wide variety of plant food and are excellent hunters of small animals such as insects, which often move quickly (Oonincx, van Leeuwen, Hendriks, \& van der Poel, 2015). Lateralization in eye use has been shown to improve hunting in other lizard species, which predate on similar types of prey (Bonati \& Csermely, 2011; Bonati et al., 2008; Robins et al., 2005). Therefore, our experiment was designed to investigate (a) whether the response elicited by a female conspecific would be similar to that towards males, as seen in previous research (Hews et al., 2004; Hews \& Worthington, 2001), and (b) what level of information is required to elicit any eye preferences. We predicted a right eye preference towards prey if videos are perceived the same way as live stimuli; a left eye preference when observing female conspecifics, if there is no difference in response towards males and females; and no eye preferences for female conspecifics if the response towards males is caused by the potential for agonistic interaction with them.

\section{Method}

\section{Subjects}

Ten adult ( 3 males and 7 females, all $>$ one year of age) and nine sub-adults ( 4 males and 5 females, ca. eight months of age) bearded dragons were used in this experiment. All animals were habituated to being handled by humans on a daily basis. They were housed in groups of 2 to 3 animals per vivarium, and males were not kept with other males to avoid aggression. The room temperature was kept at $28^{\circ} \mathrm{C}$ with additional heat lamps being provided in each vivarium. All vivaria contained UV bulbs. All animals received water ad libitum, vegetables and fruit once per day and live food (crickets and locusts) 3 times per week. 


\section{Apparatus}

The experiment took place in a rectangular arena $(120 \mathrm{~cm} \mathrm{x} 40 \mathrm{~cm})$ that was separated into two equal compartments by an opaque screen (each compartment measured $60 \mathrm{~cm} \mathrm{x} 40 \mathrm{~cm}$; Figure 1). At testing, the animals were placed into one compartment, whilst the other compartment held a computer monitor which was used to present the stimuli. The screen separating the two compartments contained a horizontal rectangular hole $(3 \mathrm{~cm} \times 5 \mathrm{~cm})$ through which the animals could see the computer monitor. This peephole constrained the bearded dragons in such a way that made it difficult for the animals to use both eyes simultaneously, making it more likely that they would view the stimuli with only the left or the right eye. It also allowed accurate coding of which eye they were using.

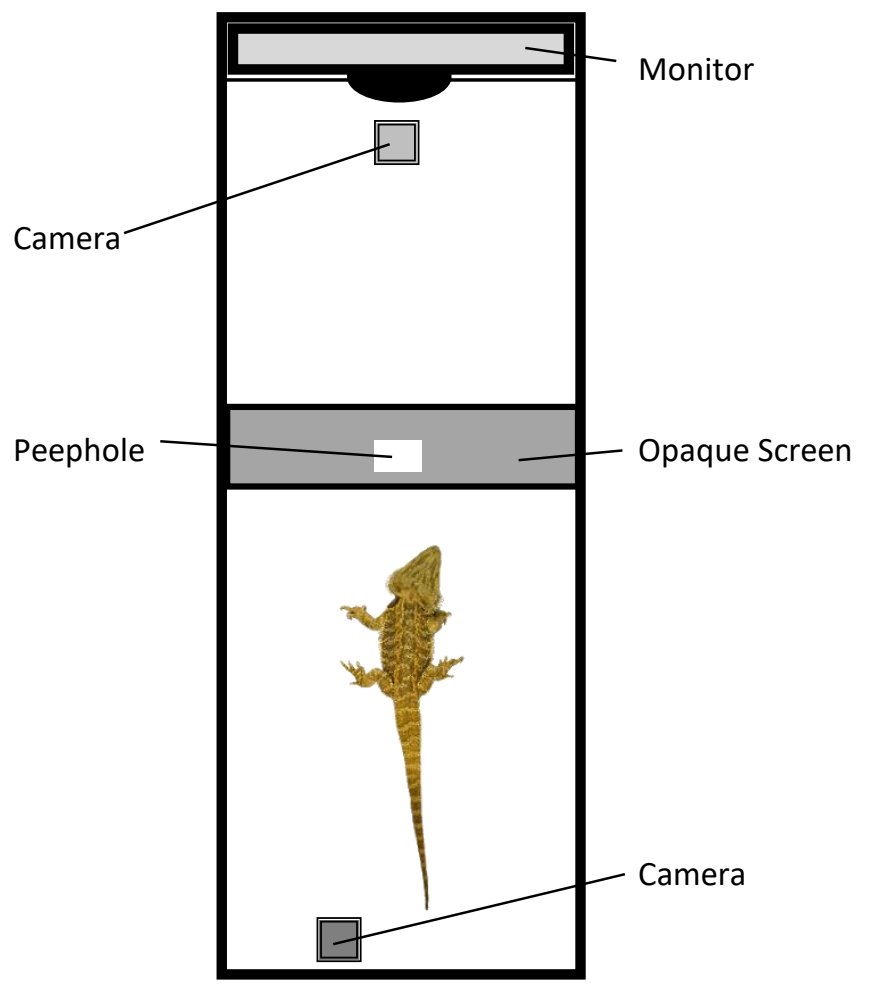

Figure1. Experimental setup, consisting of a rectangular arena, a computer monitor and an opaque screen with a peephole.

\section{Design and Procedure}

All animals were habituated to the experimental arena before starting the experiment. They were considered habituated when they readily explored and ate live food inside the arena. Each animal was given one trial per day. Each trial consisted of the presentation of one video. The videos were presented using Microsoft PowerPoint. A black screen was displayed while the bearded dragon was placed in the arena. Animals were placed in the arena facing backwards, away from the peephole. As soon as they turned around and reached the starting position (indicated by markers on the floor of the arena) the video was started manually by the experimenter. Each trial was terminated after the video ended or if the animal failed to reach the starting position within three min. Trials in which the animals failed to reach the starting position or in which the animals observed the stimulus for less than three second were discarded and repeated the following day until all animals finished all trials.

Each stimulus was a $15 \mathrm{~s}$ video belonging to one of five conditions: stationary prey (either one cricket or one locust, sitting still), moving prey (either a cricket or a locust, running or jumping), stationary conspecific (a female lizard, sitting still), moving conspecific (a female lizard, walking), and an 
empty background that was used as the control. Each animal was shown three different videos for each condition, and received a total of 15 trials. Four different videos were created per condition to avoid showing animals videos of themselves or highly familiar individuals. Therefore, the video stimuli were allocated to the individuals in a pseudo-random fashion. The order in which the stimuli were presented was also pseudo-randomized, so that no stimuli of the same condition were presented on consecutive days. The order in which the animals were tested was randomized.

The bearded dragons' behavior was recorded using two cameras (Figure 1). A small camera that was installed in front of the monitor recorded the peephole and allowed us to determine precisely which eye they were using, and an overhead webcam was used to record their position, behaviors and head movements.

\section{Data Analysis}

All recordings were analyzed using Solomon Coder (@ András Péter; version beta 15.11.19) to the nearest second. Observed behaviors included the time spent looking with the left eye, the time spent looking with the right eye, and the total amount of time animals spent looking with either (or both) eyes. Coding was blind to the testing condition. Ten percent of videos were coded for eye use (left, right or both eyes) by a second observer and inter-observer reliability was highly consistent (Spearman's correlation: $\left.r_{s}=0.93, p<0.001\right)$.

The relative use of the left eye over the right eye was compared between trials using a generalized linear mixed model (using the glmer function in the lme4 package for R version 3.3.1; Bates, Mächler, Bolker, \& Walker, 2015), with the time spent using the left eye given the time using the right eye as the binomial response variable, experimental condition (moving or stationary), stimulus species (conspecific or prey) and sex (and all three-and two-way interactions between them) as fixed factors, and individual identity as a random effect to control for the repeated use of subjects between trials. We also included an observation-level random effect term to control for overdispersion (Harrison, 2015). Significance was assessed by comparing the full model to null models lacking the fixed effect of interest using likelihood ratio tests (Crawley, 2011). We also tested whether the proportion of eye use during each condition differed from chance (i.e., a proportion of 0.5) using one-sample t-tests.

Total time spent looking at videos was compared between trials using a linear mixed-effects model (using the lme function in the nlme package), with log-transformed looking time as the response variable, fixed factors of experimental condition, stimulus species and sex (and their interaction), and individual identity as a random effect. Significance was assessed using F-tests. Comparisons with the empty background control were made using mixed models, as described above, but with only a subset of the relevant data.

We used the marginal coefficient of variation $\left(R^{2}\right)$ as a measure of effect size, calculated using the r.squaredGLMM function in the MuMIn package (Bartoń, 2016; Nakagawa \& Schielzeth, 2013).

\section{Results}

Animals looked at the videos on each trial, and spent an average of $11.21( \pm 0.27) \mathrm{s}$ per trial observing the videos. This made up $74.8 \%$ of the entire presentation time. The lizards did not frequently switch between eyes and only did so an average of $1.8 \pm 0.1$ times per trial.

There was no significant three-way interaction for lateralized eye use between sex, condition (moving or stationary) and stimulus species (conspecific or prey), $\chi^{2}(1)<0.01, p>0.99$. There were, however, significant two-way interactions between sex and stimulus species, $\chi^{2}(1)=11.19, p<0.001, R^{2}$ $=0.16$, sex and condition, $\chi^{2}(1)=10.96, p<0.001, R^{2}=0.17$, and condition and stimulus species, $\chi^{2}(1)=$ $11.21, p<0.001, R^{2}=0.17$. These interactions are detailed below.

Females used their left eye significantly longer than males when viewing conspecifics, $\chi^{2}(1)=$ $24.69, p<0.001, R^{2}=0.17$, although there was no significant difference between the sexes when viewing prey, $\chi^{2}(1)=0.58, p=0.45$. Females also showed a significantly higher proportion of left eye use when 
viewing conspecifics than prey, $\chi^{2}(1)=30.54, p<0.001, R^{2}=0.14$, although there was no significant difference for males, $\chi^{2}(1)=0.02, p=0.89$ (Figure 2A).

Females used their left eye significantly longer when looking at moving than stationary stimuli, $\chi^{2}(1)=26.66, p<0.001, R^{2}<0.01$, but there was no difference for males, $\chi^{2}(1)=1.49, p=0.22$. There was also no difference in relative eye use between females and males for stationary, $\chi^{2}(1)=2.18, p=$ 0.14 , or moving stimuli, $\chi^{2}(1)=0.19, p=0.66$ (Figure 2B).

Animals of both sexes used their left eye significantly more when viewing moving conspecific stimuli than stationary conspecific stimuli, $\chi^{2}(1)=12.72, p<0.001, R^{2}=0.16$, and moving prey stimuli compared to stationary prey, $\chi^{2}(1)=19.31, p<0.001, R^{2}=0.18$. There was no significant difference between stimulus species when they were both moving, $\chi^{2}(1)=0.02, p=0.88$, or both were stationary, $\chi^{2}(1)=2.44, p=0.12$ (Figure 2C).

For the total amount of time the animals spent looking at the stimuli with the left eye, right eye or both eyes, there was no significant three- or two-way interactions between sex, condition and stimulus species (all $p>0.13$ ). However, animals looked significantly longer at conspecific stimuli compared to prey stimuli, $F(1,169)=7.63, p=0.006, R^{2}=0.04$ (Figure 3), although there was no difference in looking time between males and females (mean \pm SE, males: $12.0 \pm 0.4 \mathrm{~s}$; females, $10.8 \pm 0.4 \mathrm{~s}$ ), $F(1,17)=2.66, p$ $=0.12$, or between moving and stationary stimuli (moving: $11.6 \pm 0.4 \mathrm{~s}$; stationary, $11.1 \pm 0.4 \mathrm{~s}$ ), $F(1,169)=0.29, p=0.59$. When compared to the empty background, animals looked longer at conspecifics (background, $10.7 \pm 0.6 \mathrm{~s}$ ), $F(1,124)=4.54, p=0.035, R^{2}=0.03$, but not prey, $F(1,125)=$ $0.03, p=0.86$ (Figure 3).

\section{Discussion}

In contrast to previous research, which focused on the perception of male lizards in videos mostly by other males (Clark et al., 1997; Macedonia \& Stamps, 1994; Ord \& Evans, 2002; Ord et al., 2002; Van Dyk \& Evans, 2007; Yang et al., 2001), this study looked at the perception of females. While it is unclear whether this species can tell the sex of conspecifics from videos, we found that even without strong visual signals typically exhibited by males, such as head bob displays, bearded dragons responded to videos of conspecifics, ruling out the possibility of them recognizing conspecifics only by this characteristic movement. Our results therefore add to the growing evidence that lizards do perceive conspecifics shown in videos as conspecifics.

We found differences between male and female lizards, with females showing a stronger left eye preference for conspecific stimuli than males, a left eye preference for moving stimuli compared to stationary ones and a left eye preference towards conspecific stimuli compared to prey. Furthermore, there was a general increase in left eye use for moving stimuli irrelevant of whether the stimuli were conspecifics or prey. There was also no difference between males and females in how much attention (measured by time spent looking) they paid to the stimuli, with all animals paying more attention to conspecifics than to prey.

There was a clear difference in how male and female lizards responded to the conspecific stimuli; females showed a significant left eye preference when observing conspecifics whilst males did not. Previous studies investigating lateralized behavior towards conspecifics have presented male stimuli (Hews et al., 2004; Hews \& Worthington, 2001) and revealed a left eye preference towards conspecifics. In this experiment, we presented female stimuli to observers of both sexes but only saw a left eye preference in the female observers. This suggests that underlying eye preference, and resultant processing, in lizards may be more complex than previously thought and different contexts may differentially elicit left eye preference in the two sexes.

The role that the behavioral ecology of the species being tested plays on responses remains unclear. However, it offers some potential for interpretation. There is evidence that the left eye/right hemisphere is responsible for the recognition of familiar conspecifics in domestic chicks. Female chicks show preferences for familiar conspecifics, while males often prefer strangers (Vallortigara \& Andrew, 1991, 1994). Bearded dragons are territorial, and unfamiliar conspecifics often display aggression towards 
Frohnwieser et al. 345

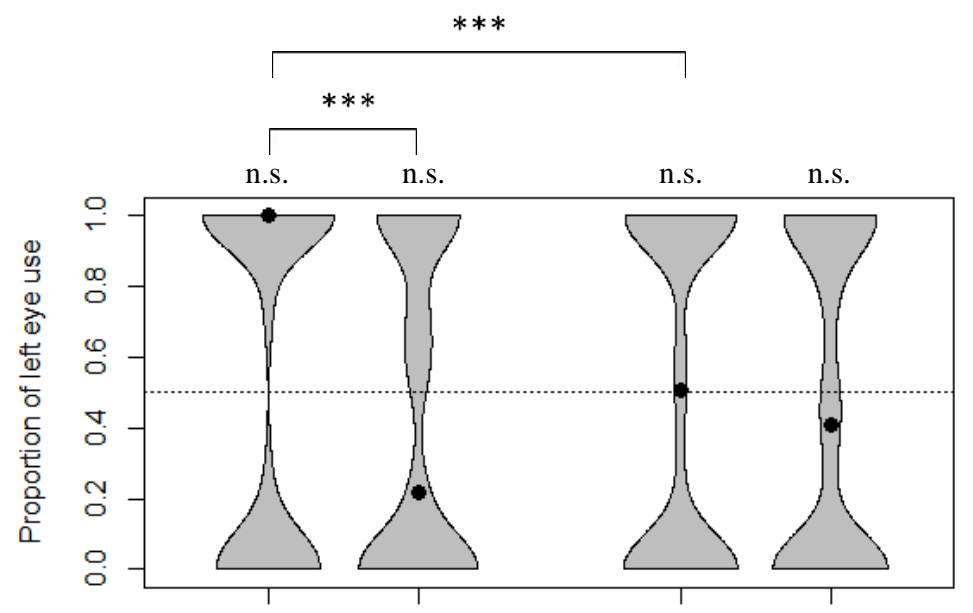

a) Female Male Conspecific

Female Male
Prey

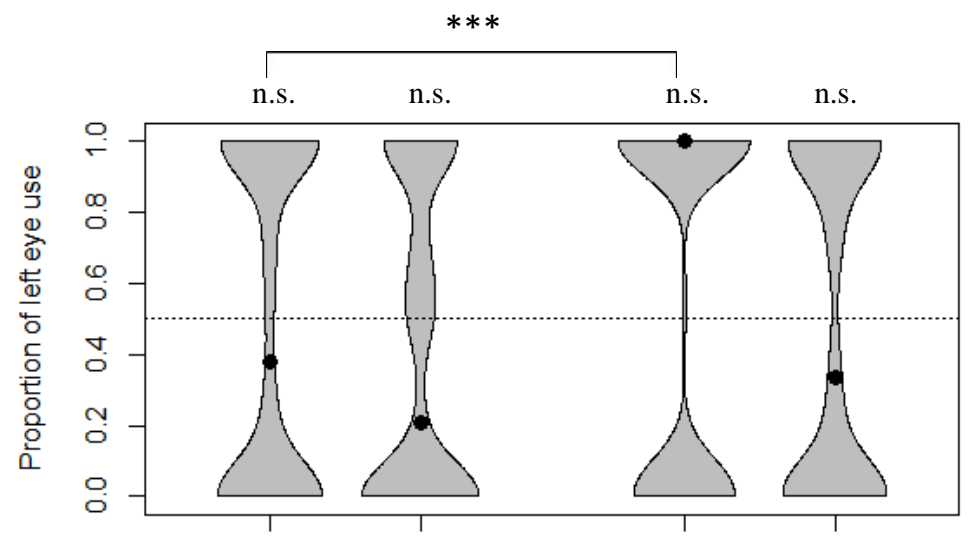

b) Female Male Stationary

Male Moving

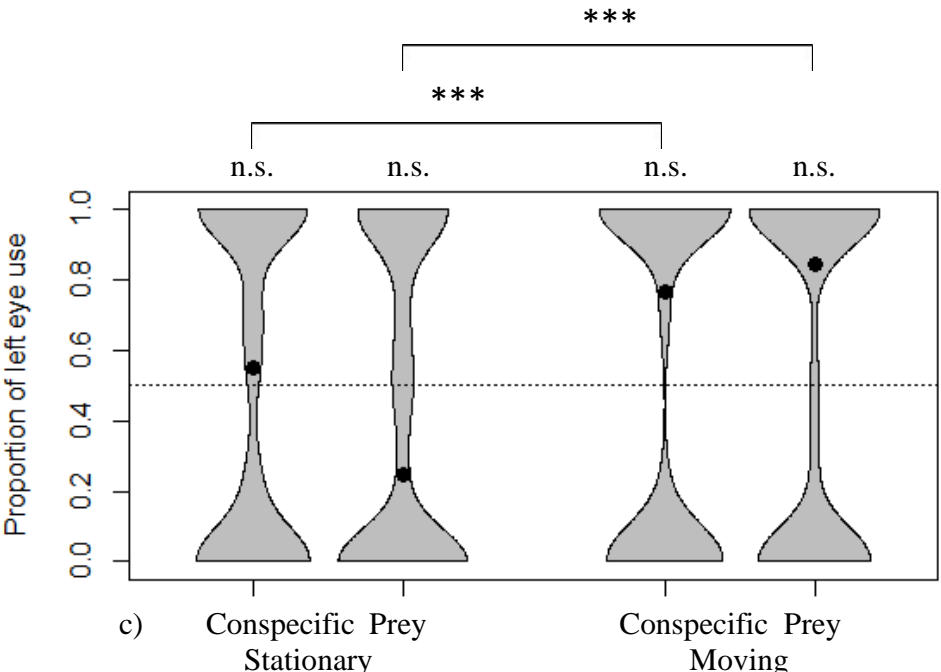

Figure 2. Proportion of left eye use for different stimuli. Beanplots (Kampstra, 2008) showing the proportion of left eye use as a function of (A) stimulus species (conspecific or prey) and sex, (B) condition (moving or stationary) and sex, and (C) condition and stimulus species. Shaded areas show the estimated density of the underlying distributions, black dots indicate medians, and the horizontal dashed line indicates chance levels of eye use. Asterisks above the boxes indicate differences from chance; lines above bars indicate significant pairwise differences: $* * * p<0.001$; n.s. non-significant. 


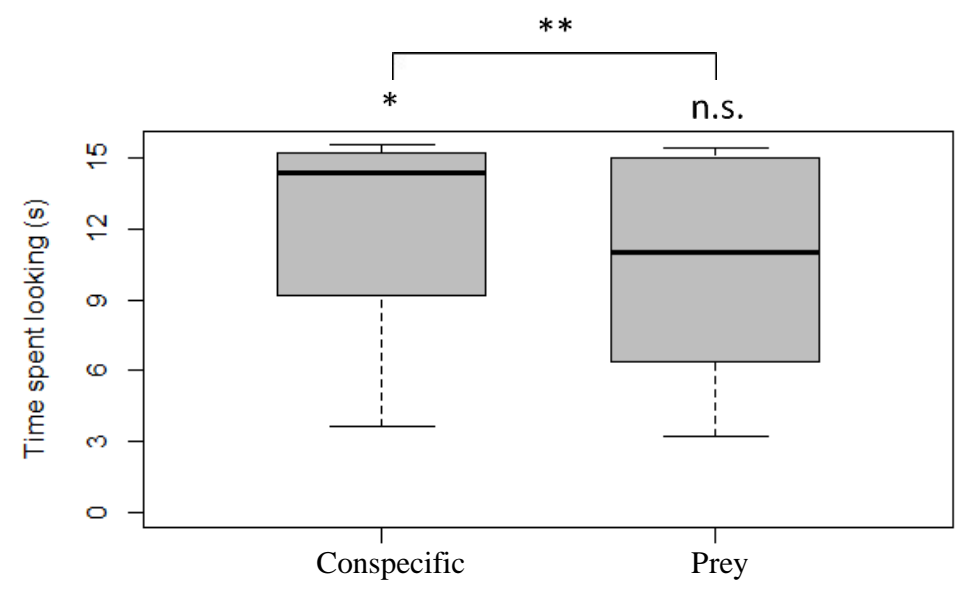

Figure 3. Amount of time spent looking at conspecific and prey stimuli. Asterisks above the boxes indicate differences from the empty background (control) trials; line above bars indicates significant differences between stimulus species: $* p<0.05$; $* * p<$ $0.01 ;$ n.s. non-significant.

each other. Unfamiliar conspecifics might pose a greater threat to females than to males, as females are generally smaller and therefore more vulnerable to attacks. Therefore, use of the left eye over the right eye may enhance females' ability to distinguish familiar from unfamiliar conspecifics. For future studies, it would therefore be interesting to compare lateralized eye use towards familiar and unfamiliar conspecifics, and test their ability to distinguish familiar and unfamiliar conspecifics with either eye. It is also possible that the findings may be the result of same-sex competition; however, more work is required before this can be ascertained. It is clear, however, that the difference was not controlled by attention, as we found no difference in the amount of time that males and females looked at the conspecific videos. Further, lizards looked at the videos depicting conspecifics longer than those depicting prey, suggesting that they were processing information from these stimuli.

While we expected a left eye preference for videos of conspecifics (Hews et al., 2004; Hews \& Worthington, 2001), it was surprising to find preferential left eye use for videos of moving prey. Most research shows right eye preferences for prey stimuli (Bonati \& Csermely, 2011; Robins et al., 2005; Vallortigara et al., 1998). However, the difference in eye use between stationary and moving stimuli suggests that this finding is likely to be an effect of movement. Prey movement has been shown to directly influence predatory behavior in lizards (Hoese, Peters, \& Evans, 2008). Though bearded dragons do eat stationary plant food, they respond very strongly to prey movement. It is therefore possible that these stimuli might have been perceived and processed differently when moving. Previous studies which investigate lateralized eye use in lizards towards prey used meal worms as stimuli (Bonati \& Csermely, 2011; Bonati et al., 2008; Robins et al., 2005). Mealworms move in a very different way to crickets and locusts and thus the impact of motion may not have previously been observed. Further work on prey perception should investigate the role of prey type and motion on stimulus perception.

In summary, this study provides important insights into the role of lateralized processing in lizard perception and sets the scene for future work investigating the role of sex on perception of conspecifics and the role of motion in lateralized eye use.

\section{References}

Bartoń, K. A. (2016). MuMIn: Multi-model inference. R package version 1.15.6. https://cran.rproject.org/web/packages/MuMIn/MuMIn.pdf

Bates, D., Mächler, M., Bolker, B. M., \& Walker, S. C. (2015). Fitting linear mixed-effects models using lme4. Journal of Statistical Software, 67, 1-48. https://doi.org/10.18637/jss.v067.i01 
Bisazza, A., De Santi, A., \& Vallortigara, G. (1999). Laterality and cooperation: Mosquitofish move closer to a predator when the companion is on their left side. Animal Behaviour, 57, 1145-1149. https://doi.org/10.1006/anbe.1998.1075

Bisazza, A., Facchin, L., Pignatti, R., \& Vallortigara, G. (1998). Lateralization of detour behaviour in poeciliid fish: The effect of species, gender and sexual motivation. Behavioural Brain Research, 91, 157-164. https://doi.org/10.1016/S0166-4328(97)00114-9

Bonati, B., \& Csermely, D. (2011). Complementary lateralisation in the exploratory and predatory behaviour of the common wall lizard (Podarcis muralis). Laterality: Asymmetries of Body, Brain and Cognition, 16, 462470. https://doi.org/10.1080/13576501003762766

Bonati, B., Csermely, D., López, P., \& Martín, J. (2010). Lateralization in the escape behaviour of the common wall lizard (Podarcis muralis). Behavioural Brain Research, 207, 1-6. https://doi.org/10.1016/j.bbr.2009.09.002

Bonati, B., Csermely, D., \& Romani, R. (2008). Lateralization in the predatory behaviour of the common wall lizard (Podarcis muralis). Behavioural Processes, 79, 171-174. https://doi.org/10.1016/j.beproc.2008.07.007

Bonati, B., Csermely, D., \& Sovrano, V. A. (2013). Looking at a predator with the left or right eye: Asymmetry of response in lizards. Laterality, 18, 329-339. https://doi.org/10.1080/1357650X.2012.673623

Braccini, S. N., Lambeth, S. P., Schapiro, S. J., \& Fitch, W. T. (2012). Eye preferences in captive chimpanzees. Animal Cognition, 15, 971-978. https://doi.org/10.1007/s10071-012-0523-9

Clark, D. L., Macedonia, J. M., \& Rosenthal, G. G. (1997). Testing video playback to lizards in the field. Copeia, 1997, 421-423. https://doi.org/10.2307/1447764

Crawley, M. J. (2011). Statistics: An introduction using R. Chichester, UK: John Wiley \& Sons Ltd.

Ghirlanda, S., \& Vallortigara, G. (2004). The evolution of brain lateralization: A game-theoretical analysis of population structure. Proceedings of the Royal Society of London B: Biological Sciences, 271, 853-857. https://doi.org/10.1098/rspb.2003.2669

Guo, K., Meints, K., Hall, C., Hall, S., \& Mills, D. (2009). Left gaze bias in humans, rhesus monkeys and domestic dogs. Animal Cognition, 12, 409-418. https://doi.org/10.1007/s10071-008-0199-3

Harrison, X. A. (2015). A comparison of observation-level random effect and Beta-Binomial models for modelling overdispersion in Binomial data in ecology \& evolution. PeerJ, 3, e1114. https://doi.org/10.7717/peerj.1114

Hews, D. K., Castellano, M., \& Hara, E. (2004). Aggression in females is also lateralized: Left-eye bias during aggressive courtship rejection in lizards. Animal Behaviour, 68, 1201-1207. https://doi.org/10.1016/j.anbehav.2003.11.024

Hews, D. K., \& Worthington, R. A. (2001). Fighting from the right side of the brain: Left visual field preference during aggression in free-ranging male tree lizards (Urosaurus ornatus). Brain, Behavior and Evolution, 58, 356-361. https://doi.org/10.1159/000057576

Hoese, F., Peters, R. A., \& Evans, C. S. (2008). The effect of variation in prey movement on the predatory response of Jacky lizards (Amphibolurus muricatus). Ethology, 114, 718-727. https://doi.org/10.1111/j.14390310.2008.01517.x

Kampstra, P. (2008). Beanplot: A boxplot alternative for visual comparison of distributions. Journal of Statistical Software, 28, 1-9. https://doi.org/10.18637/jss.v028.c01

Karenina, K. A., Giljov, A. N., \& Malashichev, Y. B. (2013). Eye as a key element of conspecific image eliciting lateralized response in fish. Animal Cognition, 16, 287 - 300. https://doi.org/10.1007/s10071-012-0572-0

Kis, A., Huber, L., \& Wilkinson, A. (2015). Social learning by imitation in a reptile (Pogona vitticeps). Animal Cognition, 18, 325-331. https://doi.org/10.1007/s10071-014-0803-7

Macedonia, J. M., \& Stamps, J. A. (1994). Species recognition in Anolis grahami (Sauria, Iguanidae): Evidence from responses to video playbacks of conspecific and heterospecific displays. Ethology, 98, 246-264. https://doi.org/10.1111/j.1439-0310.1994.tb01074.x

Martinho, A., Burns, Z. T., Von Bayern, A. M. P., \& Kacelnik, A. (2014). Monocular tool control, eye dominance, and laterality in New Caledonian crows. Current Biology, 24, 2930-2934. https://doi.org/10.1016/j.cub.2014.10.035

McKenzie, R., Andrew, R. J., \& Jones, R. B. (1998). Lateralization in chicks and hens: New evidence for control of response by the right eye system. Neuropsychologia, 36, 51-58. https://doi.org/10.1016/S00283932(97)00108-5

Nakagawa, S., \& Schielzeth, H. (2013). A general and simple method for obtaining $\mathrm{R}^{2}$ from generalized linear mixed-effects models. Methods in Ecology and Evolution, 4, 133-142. https://doi.org/10.1111/j.2041210x.2012.00261.x

Oonincx, D. G. A. B., van Leeuwen, J. P., Hendriks, W. H., \& van der Poel, A. F. B. (2015). The diet of free- 
roaming Australian central bearded dragons (Pogona vitticeps). Zoo Biology, 34, $271-277$. https://doi.org/10.1002/zoo.21209

Ord, T. J., \& Evans, C. S. (2002). Interactive video playback and opponent assessment in lizards. Behavioural Processes, 59, 55 - 65. https://doi.org/10.1016/S0376-6357(02)00045-1

Ord, T. J., Peters, R. A., Evans, C. S., \& Taylor, A. J. (2002). Digital video playback and visual communication in lizards. Animal Behaviour, 63, 879-890. https://doi.org/10.1006/anbe.2001.1983

Racca, A., Guo, K., Meints, K., \& Mills, D. S. (2012). Reading faces: Differential lateral gaze bias in processing canine and human facial expressions in dogs and 4-year-old children. PLoS ONE, 7, 1-10. https://doi.org/10.1371/journal.pone.0036076

Robins, A., Chen, P., Beazley, L., \& Dunlop, S. (2005). Lateralized predatory responses in the ornate dragon lizard (Ctenophorus ornatus). NeuroReport, 16, 849-852.

Rogers, L. J. (2012). The two hemispheres of the avian brain: Their differing roles in perceptual processing and the expression of behavior. Journal of Ornithology, 153(Suppl 1), 61-74. https://doi.org/10.1007/s10336-0110769-z

Rogers, L. J., Vallortigara, G., \& Andrew, R. J. (2013). Divided brains: The biology and behaviour of brain asymmetries. Cambridge, UK: Cambridge University Press. https://doi.org/10.1017/CBO9780511793899

Rowland, M. (2009). Veterinary care of bearded dragons. In Practice, 31, 506-511. https://doi.org/10.1136/inpract.31.10.506

Smith, A. V., Proops, L., Grounds, K., Wathan, J., \& McComb, K. (2016). Functionally relevant responses to human facial expressions of emotion in the domestic horse (Equus caballus). Biology Letters, 12, 20150907. https://doi.org/10.1098/rsbl.2015.0907

Vallortigara, G., \& Andrew, R. J. (1991). Lateralization of response by chicks to change in a model partner. Animal Behaviour, 41, 187-194. https://doi.org/10.1016/S0003-3472(05)80470-1

Vallortigara, G., \& Andrew, R. J. (1994). Differential involvement of right and left hemisphere in individual recognition in the domestic chick. Behavioural Processes, 33, 41-57. https://doi.org/10.1016/03766357(94)90059-0

Vallortigara, G., Chiandetti, C., \& Sovrano, V. A. (2011). Brain asymmetry (animal). Wiley Interdisciplinary Reviews: Cognitive Science, 2, 146-157. https://doi.org/10.1002/wcs.100

Vallortigara, G., \& Rogers, L. (2005). Survival with an asymmetrical brain: Advantages and disadvantages of cerebral lateralization. Behavioral and Brain Sciences, 28, 575-633. https://doi.org/10.1017/S0140525X05000105

Vallortigara, G., Rogers, L. J., Bisazza, A., Lippolis, G., \& Robins, A. (1998). Complementary right and left hemifield use for predatory and agonistic behaviour in toads. Neuroreport, 9, 3341-3344. https://doi.org/10.1097/00001756-199810050-00035

Van Dyk, D. A., \& Evans, C. S. (2007). Familiar-unfamiliar discrimination based on visual cues in the Jacky dragon, Amphibolurus muricatus. Animal Behaviour, 74, 33-44. https://doi.org/10.1016/j.anbehav.2006.06.018

Ventolini, N., Ferrero, E. A., Sponza, S., Della Chiesa, A., Zucca, P., \& Vallortigara, G. (2005). Laterality in the wild: Preferential hemifield use during predatory and sexual behaviour in the black-winged stilt. Animal Behaviour, 69, 1077-1084. https://doi.org/10.1016/j.anbehav.2004.09.003

Versace, E., Morgante, M., Pulina, G., \& Vallortigara, G. (2007). Behavioural lateralization in sheep (Ovis aries). Behavioural Brain Research, 184, 72-80. https://doi.org/10.1016/j.bbr.2007.06.016

Yang, E.-J., Phelps, S. M., Crews, D., \& Wilczynski, W. (2001). The effects of social experience on aggressive behavior in the green anole lizard (Anolis carolinensis). Ethology, 107, 777-793. https://doi.org/10.1046/j.1439-0310.2001.00704.x

Zucca, P., \& Sovrano, V. A. (2008). Animal lateralization and social recognition: Quails use their left visual hemifield when approaching a companion and their right visual hemifield when approaching a stranger. Cortex, 44, 13-20. https://doi.org/10.1016/j.cortex.2006.01.002 